\title{
Adjuvant Small Pelvic Radiotherapy in Patients with Cervical Cancer Having Intermediate Risk Factors Only - Is It Sufficient?
}

\author{
Sezin Yuce Sari ${ }^{\mathrm{a}}$ Ozan C. Guler ${ }^{\mathrm{b}} \quad$ Melis Gultekin $^{\mathrm{a}}$ Huseyin C. Onal ${ }^{\mathrm{c}}$ Ferah Yildiz $^{\mathrm{a}}$ \\ a Department of Radiation Oncology, Hacettepe University, Faculty of Medicine, Ankara, Turkey; \\ ${ }^{b}$ Department of Radiation Oncology, Faculty of Medicine, Karadeniz Technical University, Trabzon, Turkey; \\ ${ }^{\mathrm{c}}$ Department of Radiation Oncology, Dr Turgut Noyan Research and Treatment Center, \\ Baskent University Faculty of Medicine, Adana, Turkey
}

\section{Keywords}

Cervical cancer - Prognostic factor .

External beam radiotherapy

\section{Summary}

Background: We sought to determine the outcomes of adjuvant small pelvic external beam radiotherapy (EBRT) and prognostic factors for survival and disease control. Patients and Methods: We retrospectively evaluated 113 cervical cancer patients treated with postoperative median 50.4-Gy small pelvic EBRT. We treated the surgical bed, bilateral parametria, paravaginal soft tissues, upper third of the vagina, and presacral lymphatics. Results: Median follow-up of all patients and survivors was 58 and 67 months, respectively. The 2- and 5-year overall survival (OS) and disease-free survival rates were 91 and $82 \%$, and 85 and $74 \%$, respectively. The locoregional failure rate was $10 \%$. Age was a significant predictor for OS and distant metastasis-free survival (DMFS) on univariate analysis. The number of dissected lymph nodes being $<30$ negatively affected the pelvic recurrence-free survival. The only independent predictor on multivariate analysis was older age for DMFS. Although no severe acute toxicity was observed, late grade $\geq 3$ toxicity developed in 8 patients. Conclusion: Small pelvic EBRT produces satisfactory survival and locoregional control with acceptable toxicity, and can be an alternative to whole pelvic EBRT in selected cervical cancer patients.

(c) 2017 S. Karger GmbH, Freiburg

\section{Introduction}

Cervical cancer is the most common gynecologic cancer worldwide [1]. In International Federation of Gynecology and Obstetrics (FIGO; revised 2009) stages I-IIA, either surgery or radiotherapy (RT) can be administered with similar rates of disease control, survival, and toxicity. However, the combination of surgery and RT increases the complication rates [2-4].

A number of risk factors have been described for recurrence after surgery, i.e., parametrial invasion, compromised surgical margins, regional lymph node (LN) metastasis, unfavorable histology, large tumor, deep (> 50\%) stromal invasion (DSI), and lymphovascular space involvement (LVSI) [5-8]. In this case, adjuvant RT or chemoradiotherapy can be beneficial $[2,9]$. However, postoperative RT fields and treatment volumes are still a matter of debate. The Radiation Therapy Oncology Group (RTOG) recommends delineating the uterine surgical bed, bilateral parametria, upper third of the vagina, paravaginal soft tissues, and common, external, and internal iliac lymph nodes, and presacral lymphatics [10]. However, this field encompasses a large portion of the intestinal segments, and can cause increased toxicity. Therefore, several attempts to reduce the RT field have been made. In a pilot study by Kridelka et al. [11] 'small pelvic' RT produced high local control rates with less toxicity. Based on this trial, in 2001, we began treating all cervical cancer patients with intermediate risk factors using small pelvic external beam RT (EBRT) including the surgical bed, parametria, upper vagina, and presacral lymphatics.

In this bi-institutional study, we retrospectively evaluated the treatment outcomes and prognostic factors for survival and disease control in cervical cancer patients treated with postoperative small pelvic EBRT. The toxicity of this treatment field was also evaluated. 


\section{Patients and Methods}

The medical charts of 113 patients with early-stage intermediate-risk cervical cancer treated between 2001 and 2014 in 2 departments were retrospectively evaluated. All patients underwent pretreatment staging involving history, physical examination, vaginal examination under general anesthesia, hematologic and biochemical parameters, chest X-ray, and computed tomography (CT) or magnetic resonance imaging (MRI) of the pelvis with/without cystoscopy, rectoscopy, and intravenous pyelogram. Patients were eligible if adequate complete surgical staging was performed. Adequate LN dissection was described as the bilateral dissection of $\geq 10$ obturator and external iliac LNs [12]. Patients with $\geq 2$ intermediate risk factors (i.e., adenocarcinoma, DSI, LVSI, and $>4 \mathrm{~cm}$ tumor) or DSI alone were included in the study. All patients were treated with small pelvic EBRT. 39 (35\%) patients received additional brachytherapy, and 44 (39\%) received concurrent chemotherapy. Patients with distant metastasis or high-risk factors (i.e., positive surgical margins, LNs and/or parametria) and patients that received chemotherapy prior to RT were excluded from the study. This study was approved by the institutional review board.

All patients received either 2-dimensional (2D) or 3D conformal EBRT in supine position with a comfortably full urinary bladder and an empty rectum according to RTOG recommendations (RTOG 0418: A Phase II Study of IMRT to the Pelvis +/- Chemotherapy for Post-operative Patients with either Endometrial or Cervical Carcinoma; 2011). EBRT was administered via Philips SL25 (Philips Healthcare, Andover, MA, USA), Elekta Synergy Platform (Elekta AB, Stockholm, Sweden), or Varian Clinac DHX High Performance (Varian Medical Systems, Inc. Palo Alto, CA). In 2D EBRT, the superior border of the RT field was the lumbar (L)5-sacral (S)1 vertebral interspace, the inferior border was inferior to the obturator foramina, and the lateral borders were adjacent to the medial border of iliac bones. For lateral fields, the anterior border was posterior to the symphysis pubis, and the posterior border was the S3-4 vertebral interspace. In 3D EBRT, the surgical bed and presacral lymphatics were delineated according to the RTOG guidelines [10]. Common, internal, and external iliac LNs as well as obturator LNs were excluded. Organs at risk were detected in related slices, and were also contoured (fig. 1). In both 2D and 3D EBRT, a pelvic box technique with 4 fields (i.e., antero-posterior, postero-anterior and 2 laterals) was applied.

Brachytherapy was performed when positive or close $(<5 \mathrm{~mm})$ surgical margins on the vagina were reported. A $2 \mathrm{D}$ and $3 \mathrm{D}$ remote after-loading technique of high-dose-rate (HDR) brachytherapy was administered via microSelectron ${ }^{\circledR}$ (Elekta AB), GammaMed Plus ${ }^{\circledR}$ (Varian Medical Systems Inc.), or VariSource $^{\circledR}$ (Varian Medical Systems Inc.). All patients were treated in supine position with an empty urinary bladder and rectum. A vaginal cylinder covering the whole vagina was placed. The dose was prescribed at a $5-\mathrm{mm}$ distance from the mucosa. In 3D brachytherapy, a planning CT with a slice thickness of $2.5 \mathrm{~mm}$ was taken, and the clinical target volume (CTV) was contoured encompassing the upper third (length) and whole thickness of the vagina.

A radiation oncologist followed all patients during the course of RT and, in association with a gynecologic oncologist, monitored patients every 3 months for the first 2 years, every 6 months until year 5, and annually thereafter. Follow-up information was obtained from department charts, referring doctors, and patients and/or next of kin. RTOG/European Organization for the Treatment of Cancer (EORTC) toxicity criteria were used to score RT-related acute and late toxicities [13].

All statistical analyses were performed using the Statistical Package for the Social Sciences (SPSS) version 18.0 (SPSS Inc., IBM Corp., Armonk, NY, USA). The primary end point was pelvic recurrence-free survival (PRFS). Secondary end points included overall survival (OS), disease-free survival (DFS), distant metastasis-free survival (DMFS), and treatment toxicity. Time to progression was calculated as the period from the date of diagnosis to the date of death or disease recurrence. Survival analyses were carried out using the Kaplan-Meier method and compared using the log-rank test. Multivariate analysis was performed using the Cox proportional hazards model, including all factors found significant by univariate analysis. $\mathrm{p}<0.05$ was considered statistically significant.

\section{Results}

Patient and treatment characteristics are presented in table 1. The median age was 54 years (range 34-84 years). Tumors were mostly squamous cell carcinoma. The median number of dissected total, pelvic, and para-aortic LNs was 41 (range 12-120), 30 (range 12-97), and 8 (range 0-36), respectively. 61 (54\%) patients received 2D EBRT, whereas $52(46 \%)$ received 3D EBRT. The median EBRT dose was 50.4 Gy (range 45-50.4 Gy) in median 1.8-Gy (range 1.8-2 Gy) daily fractions. 39 (35\%) patients underwent HDR brachytherapy with a median 15 Gy (range 12-28 Gy) in 5-Gy (range 4-7 Gy) daily fractions. 44 (39\%) patients received concurrent $40 \mathrm{mg} / \mathrm{m}^{2}$ weekly cisplatin with a median of 5 (range 3-6) cycles.

The median follow-up times for all patients and for those that survived were 58 months (range 2-177 months) and 67 months (range 4-177 months), respectively. In total, 6 patients harbored disease (local, locoregional, or distant), including 2 with distant
Fig. 1. Dose-color wash images of 3-dimentional radiotherapy plan in a cervical cancer patient with postoperative intermediate risk factors. Right upper image: right and left femur depicted in blue and pink, respectively. Dose-color wash images are seen in transverse, coronal, and sagittal slices in the upper left, lower left, and lower right boxes, respectively.

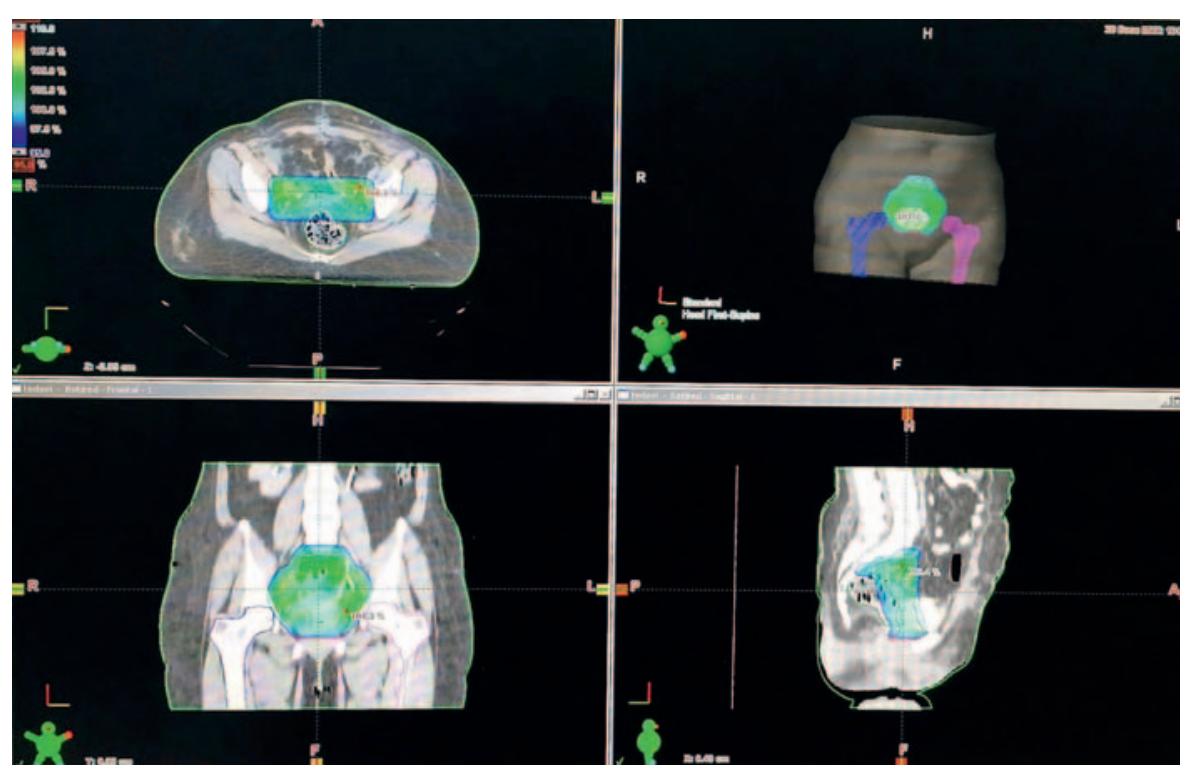


Table 1. Patient and treatment characteristics

\begin{tabular}{|c|c|}
\hline Characteristic & Patients, n (\%) \\
\hline \multicolumn{2}{|l|}{ Age, years } \\
\hline$\leq 50$ & $41(36)$ \\
\hline$>50$ & $72(64)$ \\
\hline \multicolumn{2}{|l|}{ Type of surgery } \\
\hline Type II & $4(3)$ \\
\hline Type III & $109(97)$ \\
\hline \multicolumn{2}{|l|}{ Stage } \\
\hline IA2 & $1(1)$ \\
\hline IB1 & $53(47)$ \\
\hline IB2 & $41(36)$ \\
\hline IB (tumor size not achieved) & $2(2)$ \\
\hline IIA1 & $13(11)$ \\
\hline IIA2 & $3(3)$ \\
\hline \multicolumn{2}{|l|}{ Tumor size, $\mathrm{cm}$} \\
\hline$\leq 2$ & $9(8)$ \\
\hline $2-4$ & $58(51)$ \\
\hline$>4$ & $40(36)$ \\
\hline N/A & $6(5)$ \\
\hline \multicolumn{2}{|l|}{ Histopathology } \\
\hline SCC & $98(87)$ \\
\hline Adenocarcinoma & $13(11)$ \\
\hline Adenosquamous carcinoma & $2(2)$ \\
\hline \multicolumn{2}{|l|}{ Cervical stromal invasion, \% } \\
\hline$\leq 50$ & $8(7)$ \\
\hline$>50$ & $83(74)$ \\
\hline N/A & $22(19)$ \\
\hline Endometrial invasion & $24(21)$ \\
\hline Myometrial invasion & $12(11)$ \\
\hline Lymphovascular space involvement & $56(49)$ \\
\hline \multicolumn{2}{|l|}{ Vaginal SM } \\
\hline Positive & $4(4)$ \\
\hline Close $(<0.5 \mathrm{~cm})$ & $28(25)$ \\
\hline VAIN on SM & $2(2)$ \\
\hline In-situ cervical carcinoma on SM & $1(1)$ \\
\hline \multicolumn{2}{|l|}{ Risk factors, $\mathrm{n}$} \\
\hline 1 (only DSI) & $19(17)$ \\
\hline 2 & $46(41)$ \\
\hline 3 & $23(20)$ \\
\hline N/A & $25(22)$ \\
\hline
\end{tabular}

N/A = Not available; SCC = squamous cell carcinoma; $\mathrm{SM}=$ surgical margin; VAIN = vaginal intraepithelial neoplasia; DSI = deep stromal invasion.

metastasis, 2 with pelvic recurrence, and 2 with both. At the final follow-up, 91 (81\%) patients were alive ( 9 with disease), and 22 (19\%) patients had died ( 6 of their disease, 17 of other causes).

The 2- and 5-year OS rate was 91 and $82 \%$, respectively. In univariate analysis, age was the only significant factor (table 2). In multivariate analysis, no statistically significant factor was observed. The 2- and 5-year DFS rate was 85 and 74\%, respectively. In univariate analysis, a trend for DFS was observed in patients $\leq 50$ years $(\mathrm{p}=0.09)$ and in patients with $\geq 30$ LNs dissected $(\mathrm{p}=0.06)$. No statistically significant factor was found in multivariate analysis. The 2- and 5-year PRFS rate was 86 and 75\%, respectively. The locoregional control rate was $92 \%$ in 2 years and $89 \%$ in 5 years. The
Table 2. Prognostic factors in univariate analysis for overall survival

\begin{tabular}{|c|c|c|c|}
\hline & 2-year OS, \% & 5-year OS, \% & $\mathrm{p}$ value \\
\hline \multicolumn{3}{|l|}{ Age, years } & \multirow[t]{3}{*}{$0.04^{\mathrm{a}}$} \\
\hline$\leq 50$ & 90 & 90 & \\
\hline$>50$ & 92 & 78 & \\
\hline \multicolumn{3}{|l|}{ Dissected LNs, $\mathrm{n}$} & \multirow[t]{3}{*}{0.08} \\
\hline$<30$ & 75 & 75 & \\
\hline$\geq 30$ & 92 & 83 & \\
\hline \multicolumn{3}{|l|}{ SM status } & \multirow[t]{3}{*}{0.09} \\
\hline Positive/close & 82 & 70 & \\
\hline Negative & 92 & 86 & \\
\hline \multicolumn{4}{|c|}{ a Statistically significant. } \\
\hline
\end{tabular}

2- and 5-year PRFS rates were 74 and 66\%, and 92 and $79 \%$ in patients with $<30$ and $\geq 30$ dissected LNs, respectively $(\mathrm{p}=0.04)$. Multivariate analysis was not performed as there was only 1 statistically significant factor in univariate analysis. The 2- and 5-year DMFS rate was 88 and 79\%, respectively. The distant metastasis rate was $6 \%$ for the entire cohort. The only statistically significant factor in univariate analysis was age. The 2- and 5-year DMFS rate was 90 and $88 \%$ in patients $\leq 50$ years compared to 87 and $73 \%$ in patients $>50$ years, respectively $(\mathrm{p}=0.02)$. Although not statistically significant, positive/close surgical margins on the vagina resulted in a decreased DMFS rate (2- and 5-year DMFS rate 79 and 67\% vs. 92 and $83 \%$ in patients with negative margins; $\mathrm{p}=0.075)$. Older age $(>50$ years) was the only significant factor in multivariate analysis (hazard ratio $=2.77,95 \%$ confidence interval $1.02-7.56 ; \mathrm{p}=0.04$ ).

None of the patients had toxicities that necessitated the discontinuation of RT. No grade $\geq 3$ adverse events occurred during EBRT or brachytherapy. 45 (40\%) patients developed grade 1-2 acute toxicity (cystitis, $\mathrm{n}=24$; proctitis, $\mathrm{n}=24$; diarrhea, $\mathrm{n}=21$; dermatitis, $\mathrm{n}=7$ ). Late serious complications were observed in 8 (7\%) patients: 1 patient each with proctitis, enteritis, lymphedema, rectovaginal fistula, rectovesical fistula, enterovesical fistula, femoral aseptic necrosis, and femoral aseptic necrosis with a possibly RT-related sarcoma of the iliac bone. The rate of late toxicity in patients treated with 2D EBRT was significantly higher compared to that in patients treated with 3D EBRT $(p=0.04)$. However, only 1 patient with a rectovaginal fistula was treated with $3 \mathrm{D}$ EBRT. The patient with late proctitis had received $4 \times 7$ Gy brachytherapy, and also developed grade 2 proctitis during EBRT. Other patients with late toxicity had not received brachytherapy, and none of them developed acute toxicity.

\section{Discussion}

The results of the present study revealed that omitting the iliac and obturator lymphatics during EBRT in early-stage cervical cancer patients with intermediate risk factors does not negatively impact survival and disease control. Additionally, we did not find any significant prognosticators for survival or local control, except for 
age for OS and DMFS. Older patients in our study received a quite similar treatment schedule with high compliance rates. We can only assume that the reason for the worse outcome in patients $>50$ years may be somewhat aggressive tumor factors other than the already known.

Postoperative adjuvant RT improves local control in selected cases. In the studies on postoperative RT, cervical cancer patients mostly have high and intermediate risk factors $[14,15]$. In a phase III study of patients with intermediate risk factors alone, Sedlis et al. [2] treated 277 patients with 46-50.4 Gy EBRT that encompassed the obturator and iliac LNs. With a median 10-year followup, the authors reported DFS rates of $82.5 \%$ in patients treated with adjuvant RT and $69.3 \%$ for no further treatment [16]. The locoregional recurrence rate was 13.9 and $20.7 \%$ in the respective patients. The overall pelvic disease control rate in our study was $90.3 \%$ which is quite similar to the above study with whole pelvic EBRT.

A number of studies did not recommend adjuvant RT in earlystage cervical cancer with intermediate risk factors as it did not increase disease control and survival rates [17, 18]. Nevertheless, the majority of studies has come up with opposite results. DSI and tumor size were demonstrated as strong prognostic factors for OS, DFS, and DMFS $[9,19,20]$. In the Gynecologic Oncology Group (GOG)-49 study, Delgado et al. [21] demonstrated that tumor size, LVSI, and DSI were independent predictors for DFS in stage IB disease. In the GOG-92 study with 277 patients having $\geq 2$ intermediate risk factors, compared to surgery alone, adjuvant RT including iliac and obturator LNs reduced the recurrence and mortality risk by 44 and $36 \%$, respectively [2]. In the update of this study with a median 10-year follow-up, local and distant failures were still higher in the surgery-only arm compared to the adjuvant RT arm (20.7 vs. $13.9 \%$, and 8.6 vs. $2.9 \%)$ with similar OS rates (70 vs. $81 \%$ ) [16]. Based on these findings, we began delivering adjuvant RT to all patients with DSI alone or $\geq 2$ intermediate risk factors. However, we found none of the intermediate risk factors to be a significant prognostic factor in the current study. The routine use of adjuvant RT may have precluded these risk factors and hence the determination of their effect on local and distant recurrence.

According to the American College of Radiology (ACR) Appropriateness Criteria, patients with early-stage cervical cancer are divided into 3 risk groups following radical surgery, and patients with LVSI, DSI, and/or large tumor constitute the intermediaterisk group [22]. Adjuvant RT is indicated in this group as the recurrence risk is approximately $30 \%$. For adjuvant RT, ACR recommends that the CTV should encompass the paracervical, parametrial, and paravaginal soft tissues, the upper vagina, the ligaments supporting the cervix, and all LNs at high risk of involvement [22]. In $2 \mathrm{D}$ simulation, the superior border is the L5-S1 vertebral interspace, the inferior border is inferior to the iliac bones, and the lateral borders are $2 \mathrm{~cm}$ lateral to the iliac foramina. In our small pel- vic field, the superior border is the L5-S1 vertebral interspace, but the inferior border is just inferior to the iliac foramina and the lateral borders are lateral to these. Hence, our field is relatively smaller than conventional fields. Nevertheless, only $10 \%$ of patients experienced pelvic recurrence in a median follow-up of 58 months. Our results are comparable with those of Kriedelka et al. [11] who used small pelvic fields in 25 patients with intermediate risk factors of whom only 1 patient recurred.

RTOG published a consensus guideline for IMRT in postoperative endometrial and cervical cancer [10]. They recommend delineating common, external, and internal iliac LNs as well as presacral LNs together with the uterine and cervical surgical bed, bilateral parametria, upper third of the vagina, and paravaginal soft tissues. We strictly follow this guideline when determining targets. However, we irradiate presacral lymphatics alone in patients with adequate $\mathrm{LN}$ dissection. We give an at least $1.5-\mathrm{cm}$ margin for the planning target volume based on organ motion according to our previous study with sequential CT scans at different time periods of treatment [23].

None of our patients experienced toxicity that necessitated the discontinuation of RT. Only 8 patients developed considerable late toxicity. In studies on cervical cancer patients with high and intermediate risk factors treated with adjuvant low pelvic field EBRT (i.e., exclusion of common iliac LNs), local and distant control rates were found to be similar to those in patients treated with whole pelvic RT with much lower rates of gastrointestinal complications $[15,24,25]$. In our study, delineating only the presacral LNs resulted in an excellent pelvic control rate of $90.3 \%$ compared to studies with whole pelvic EBRT $[14,26]$. A 5-year OS rate of $82 \%$ and the absence of grade $\geq 3$ acute toxicity also reveal that adjuvant small pelvic EBRT is an adequate and sufficient treatment for patients with intermediate risk factors.

The main limitations of our study are that it is retrospective in nature, and it only presents the results of small pelvic EBRT without comparing it to whole pelvic EBRT. However, this study is important in that it offers a somewhat innovative technique to radiation oncologists which leads to excellent disease control with negligible toxicity. Although there are previously published studies presenting acceptable outcomes with small pelvic EBRT, our study is the only one that applied a true small pelvic field. Besides the identified intermediate risk factors, older age and $<30 \mathrm{LN}$ dissection deteriorated the prognosis. On the other hand, prospective trials analyzing the treatment outcomes and prognostic factors for small and whole pelvic field irradiation are required for accurate decision making.

\section{Disclosure Statement}

The authors declare no conflict of interest. 


\section{References}

12012 GLOBOCAN Cancer Incidence and Mortality Worldwide. IARC CancerBase, 2010; globocan.iarc.fr/ Pages/fact_sheets_cancer.aspx.

2 Sedlis A, Bundy BN, Rotman MZ, Lentz SS, Muderspach LI, Zaino RJ: A randomized trial of pelvic radiation therapy versus no further therapy in selected patients with stage IB carcinoma of the cervix after radical hysterectomy and pelvic lymphadenectomy: a Gynecologic Oncology Group Study. Gynecol Oncol 1999; 73:177-183.

3 Landoni F, Maneo A, Colombo A, Placa F, Milani R, Perego P, Favini G, Ferri L, Mangioni C: Randomised study of radical surgery versus radiotherapy for stage Ib-IIa cervical cancer. Lancet 1997;350:535-540.

4 Gray HJ: Primary management of early stage cervical cancer (IA1-IB) and appropriate selection of adjuvant therapy. J Natl Compr Canc Netw 2008;6:47-52.

5 Horn LC, Fischer U, Raptis G, Bilek K, Hentschel B: Tumor size is of prognostic value in surgically treated FIGO stage II cervical cancer. Gynecol Oncol 2007; 107:310-315.

6 Aoki Y, Sasaki M, Watanabe M, Sato T, Tsuneki I, Aida H, Tanaka K: High-risk group in node-positive patients with stage IB, IIA, and IIB cervical carcinoma after radical hysterectomy and postoperative pelvic irradiation. Gynecol Oncol 2000;77:305-309.

7 Atahan IL, Yildiz F, Ozyar E, Pehlivan B, Genc M, Kose MF, Tulunay G, Ayhan A, Yuce K, Guler N, Kucukali T: Radiotherapy in the adjuvant setting of cervical carcinoma: treatment, results, and prognostic factors. Int J Gynecol Cancer 2007;17:813-820.

8 Nahhas WA, Sharkey FE, Whitney CW, Husseinzadeh $\mathrm{N}$, Chung CK, Mortel R: The prognostic significance of vascular channel involvement and deep stromal penetration in early cervical carcinoma. Am J Clin Oncol 1983;6:259-264.

9 Peters WA 3rd, Liu PY, Barrett RJ 2nd, Stock RJ, Monk BJ, Berek JS, Souhami L, Grigsby P, Gordon W Jr, Alberts DS: Concurrent chemotherapy and pelvic radiation therapy compared with pelvic radiation therapy alone as adjuvant therapy after radical surgery in highrisk early-stage cancer of the cervix. J Clin Oncol 2000; 18:1606-1613.
10 Small W Jr, Mell LK, Anderson P, Creutzberg C, De Los Santos J, Gaffney D, Jhingran A, Portelance L, Schefter T, Iyer R, Varia M, Winter K, Mundt AJ: Consensus guidelines for delineation of clinical target volume for intensity-modulated pelvic radiotherapy in postoperative treatment of endometrial and cervical cancer. Int J Radiat Oncol Biol Phys 2008;71:428-434.

11 Kridelka FJ, Berg DO, Neuman M, Edwards LS, Robertson G, Grant PT, Hacker NF: Adjuvant small field pelvic radiation for patients with high risk, stage IB lymph node negative cervix carcinoma after radical hysterectomy and pelvic lymph node dissection. A pilot study. Cancer 1999;86:2059-2065.

12 Nijman HW, Khalifa M, Covens A: What is the number of lymph nodes required for an 'adequate' pelvic lymphadenectomy? Eur J Gynaecol Oncol 2004;25: 87-89.

13 Cox JD, Stetz J, Pajak TF: Toxicity criteria of the Radiation Therapy Oncology Group (RTOG) and the European Organization for Research and Treatment of Cancer (EORTC). Int J Radiat Oncol Biol Phys 1995; 31:1341-1346.

14 Yeh SA, Wan Leung S, Wang CJ, Chen HC: Postoperative radiotherapy in early stage carcinoma of the uterine cervix: treatment results and prognostic factors. Gynecol Oncol 1999;72:10-15.

15 Hong JH, Tsai CS, Lai CH, Chang TC, Wang CC, Lee SP, Tseng CJ, Hsueh S: Postoperative low-pelvic irradiation for stage I-IIA cervical cancer patients with risk factors other than pelvic lymph node metastasis. Int J Radiat Oncol Biol Phys 2002;53:1284-1290.

16 Rotman M, Sedlis A, Piedmonte MR, Bundy B, Lentz SS, Muderspach LI, Zaino RJ: A phase III randomized trial of postoperative pelvic irradiation in Stage IB cervical carcinoma with poor prognostic features: followup of a gynecologic oncology group study. Int J Radiat Oncol Biol Phys 2006;65:169-176.

17 Sartori E, Tisi G, Chiudinelli F, La Face B, Franzini R, Pecorelli S: Early stage cervical cancer: adjuvant treatment in negative lymph node cases. Gynecol Oncol 2007;107:S170-174.

18 Van der Velden J, Samlal R, Schilthuis MS, Gonzalez DG, ten Kate FJ, Lammes FB: A limited role for adjuvant radiotherapy after the Wertheim/Okabayashi radical hysterectomy for cervical cancer confined to the cervix. Gynecol Oncol 1999;75:233-237.
19 Chen SW, Liang JA, Yang SN, Lin FJ: Early stage cervical cancer with negative pelvic lymph nodes: pattern of failure and complication following radical hysterectomy and adjuvant radiotherapy. Eur J Gynaecol Oncol 2004;25:81-86

20 Gadducci A, Fabrini MG, Bonuccelli A, Fanucchi A, Perutelli A, Facchini V: Recurrence patterns in patients with early stage cervical cancer treated with radical hysterectomy and external pelvic irradiation. Anticancer Res 1995;15:1071-1077.

21 Delgado G, Bundy BN, Fowler WC Jr, Stehman FB Sevin B, Creasman WT, Major F, DiSaia P, Zaino R: A prospective surgical pathological study of stage I squamous carcinoma of the cervix: a Gynecologic Oncology Group Study. Gynecol Oncol 1989;35:314-320.

22 Wolfson AH, Varia MA, Moore D, Rao GG, Gaffney DK, Erickson-Wittmann BA, Jhingran A, Mayr NA, Puthawala AA, Small W Jr, Yashar CM, Yuh W, Cardenes HR; American College of Radiology (ACR): ACR Appropriateness Criteria( $R$ ) role of adjuvant therapy in the management of early stage cervical cancer. Gynecol Oncol 2012;125:256-262.

23 Yilmaz C, Gultekin M, Eren G, Yuce D, Yildiz F: Determination of optimal planning target volume margins in patients with gynecological cancer. Phys Med 2015;31:708-713.

24 Ohara K, Tsunoda H, Satoh T, Oki A, Sugahara S, Yoshikawa H: Use of the small pelvic field instead of the classic whole pelvic field in postoperative radiotherapy for cervical cancer: reduction of adverse events. Int J Radiat Oncol Biol Phys 2004;60:258-264.

25 De Jong RA, Pras E, Boezen HM, van der Zee AG, Mourits MJ, Arts HJ, Aalders JG, Slot A, Timmer PR, Hollema H, Nijman HW: Less gastrointestinal toxicity after adjuvant radiotherapy on a small pelvic field compared to a standard pelvic field in patients with endometrial carcinoma. Int J Gynecol Cancer 2012;22 1177-1186.

26 Tsai CS, Lai CH, Wang CC, Chang JT, Chang TC, Tseng CJ, Hong JH: The prognostic factors for patients with early cervical cancer treated by radical hysterectomy and postoperative radiotherapy. Gynecol Oncol 1999; 75:328-333. 